\title{
Curtailing COVID-19 spread in drain pipelines: Using interfacial hydrodynamics for removing Bacterial and Viral Biofilms
}

\section{P P Shahabaz}

Indian Institute of Technology Bombay

Janani Srree Murallidharan ( $\boldsymbol{\nabla}$ js.murallidharan@iitb.ac.in )

Indian Institute of Technology Bombay

\section{Research Article}

Keywords: COVID-19, interfacial flows, CFD

Posted Date: August 19th, 2020

DOI: https://doi.org/10.21203/rs.3.rs-61417/v1

License: (c) (1) This work is licensed under a Creative Commons Attribution 4.0 International License.

Read Full License 


\title{
Curtailing COVID-19 spread in drain pipelines : \\ Using interfacial hydrodynamics for removing Bacterial and Viral Biofilms
}

\author{
P P Shahabaz, and Janani Srree Murallidharan* \\ *Correspondence to js.murallidharan@iitb.ac.in
}

\begin{abstract}
Drainage systems contains biological contaminants like bacteria and viruses flowing through them. Additionally, these pipelines also have organic matter known as biofilms growing on their walls. These biofilms infact act as incubation zones for further growth of bacteria and coronaviruses. Standard water treatment routines with traditional cleaning agents are known to be not be able to clean or sterilize microbes located in the inner layers of the biofilm. A recent study has identified specialised fluids which are effective in removing biofilms but these need to be used prudently. The present study proposes to use ' interfacial hydrodynamics' to ensure that the cleaner-fluid (CF) is transported effectively to the location of the biofilms at the pipe walls, and allowed to be in contact with the biofilms for a sufficient amount of time so as to ensure its effective removal. The present study has used CFD technique of Multi-fluid VOF and has demonstrated that relative superficial velocities of cleanerfluids and sewage water can be controlled, so as to achieve flow regimes that ensure delivery of cleaner fluid to the periphery of the tube walls. Our simulations indicate that most effective cleaning can be achieved by using a cleaner-fluid with a high viscosity ( $~ 5000 \mathrm{cP}))$. In such cases, a low- medium velocity $(\sim 0.05-0.3 \mathrm{~m} / \mathrm{s})$ of CF and water would ensure that the cleaner fluids are in constant contact with the pipe walls. Other suitable viscosity and velocity combinations have also been proposed. Flow parameters that can be used to monitor and cross-verify expected flow patterns on-site have also been proposed.
\end{abstract}




\title{
Curtailing COVID-19 spread in drain pipelines -
}

\section{Using interfacial hydrodynamics for removing Bacterial and Viral Biofilms}

\author{
P P Shahabaz, and Janani Srree Murallidharan* \\ Department of Mechanical Engineering, \\ Indian Institute of Technology Bombay, Mumbai 400076, India \\ *Correspondence to js.murallidharan@iitb.ac.in
}

\section{INTRODUCTION}

Most cities around the world have drainage systems to collect rain runoff, wastewater, and sewage. The 'effluent' flowing through these pipelines usually contains biological and chemical contaminants. Though, standard water treatment routines are thought sufficient to remove most of the biological contaminants in both drinking and wastewater [1], in 2003, it was a sewage leak that caused a SARS outbreak (a type of coronavirus) in Hong Kong. Worryingly, in Chennai, a densely populated metropolitan city in India, authorities have detected the presence of Coronavirus-COVID-19 RNA in sewage samples collected as part of a preliminary study[2].Several such instances have also been reported around the world. Though no known cases of COVID-19 have been caused by sewage leaks [1], COVID19 which is closely related to SARS, could also potentially cause outbreaks such as the one experienced in Hong Kong [3][4]. Though, WHO has emphasised that wastewater should be treated in well-designed and well-managed centralized wastewater treatment plants, the incidence in 2003, has clearly established that the standard treatment protocols are not sufficient to remove SARS-CoV-19 from drain lines.

Coronaviruses' survival in water is found to be dependent on: (a)Temperature (b) Light exposure (c) Organic matter (adsorption of virus onto organic matter) and (d) Presence of antagonist microorganisms [5]. Organic matter tends to act as incubation zones for coronaviruses. One such organic matter, and a potential incubation zone for coronavirus inside pipelines, are 'biofilms'. Biofilms are thin, slimy bacterial growths that line the pipes of many aging drinking water and sewage systems. These biofilms are found to release $58 \%$ of the bacteria in the distributed water [6]. Simple hydrodynamic means to prevent biofilms' growth, such as using fast flowing water, have been found ineffective [7] as biofilms can withstand high shear stresses especially in dead-ends. Traditional cleaning agents are also known to be ineffective and do not clean or sterilize microbes located in the inner layers of the biofilm [8]. A recent study has identified a class of fluids known as enzyme fluids [9][10] to be effective for removing biofilms in pipes. These enzyme fluids are specifically being highlighted here, as these fluids are organic and thus will be safe for human use. In principle, any cleaner fluid which is thought appropriate can also be used. However, any of these specialised cleaner fluids tend to be expensive and need to be used prudently. The best way to ensure minimal use of these fluids, and to achieve effective cleaning of large pipelines is to ensure that the cleaner-fluid (CF) is transported effectively to the location of the biofilms, and allowed to be in contact with the biofilms for a sufficient amount of time so as to ensure its effective removal. The present study 
proposes to use 'multiphase flow' hydrodynamics to achieve the same, and to counter COVID-19 outbreak that can originate from sewage pipelines.

\section{Concept of Flow Regimes:}

Multiphase flows have fluids interacting among themselves and result in a wide array of flow configuration formations, known as flow regimes (for example, see Table 1-3). Out of all the flow regimes possible, certain flow regimes are more suited to efficiently transport cleaner-fluids to the biofilms. The temporal and spatial distribution of these flow regimes depend on the flow rates of individual phases, the configuration of the flow domain, and also on the properties of the fluids. Flow pattern maps of liquid-liquid systems, provide this information, however such flow pattern maps available in literature are currently limited in their applicability range. This paper, with the aim of recommending best flow conditions to achieve effective cleaning, has carried out simulations of liquid-liquid systems for various parametric ranges, and has thus helped expand the applicability range.

Flow regimes have different sizes/types of interfaces. Consequently, different mathematical techniques are required to capture it accurately. Dispersed flows have been traditionally solved using Eulerian two-fluid models. On the other hand, the interface tracking models have been developed for the modelling of flows having large distinctive interfaces between the phases [11][12]. Since both methods discussed above (two fluid and interface tracking) are specific to a particular flow regime, a coupled approach for flows involving mixed regimes have been developed [13]. Here in these coupled methods, the Eulerian multi-fluid dispersion models is selected, when the interface scales are small enough to be resolved, and the tracking method is used when the interface is larger than or comparable with the grid dimension. The unified models formed by the coupling of both the models had to employ a criterion for switching between the different models [14][15][16]. Due to the complexity of the flow regimes being dealt with, and the wide range of possibilities, the coupled Multi-fluid VOF is considered the most suitable method to capture most of the flow regimes occurring in pipelines and hence is used in this study.

For the present work, using CFD simulations, we demonstrate that interfacial hydrodynamic concepts can be used to ensure precise transfer of cleaner-fluid to tube walls, and also to ensure sufficient contact, in order for proper removal of biofilms. In particular, we show that high viscosity CFs, followed by medium viscosity CFs, are most suitable for cleaning purposes. In our simulations, the low viscosity fluids, which are traditionally thought best, are largely ineffective. This unexpected result is due to the fact that high viscosity flows have the tendency to retain their interfaces against the forces exerted by surrounding water and are easily transported to the pipe wall. The theory relies on interfacial hydrodynamics driven by the difference in viscosities and relative superficial velocities, and is therefore not limited to water and a specific cleaner fluid combination. In addition, we show 
that the computational technique used here can be an effective tool that can be used to predict the flow regime formation for drain pipeline flows, for a wide range of fluid combinations. To ensure that real-life conditions mimic what is predicted by simulations, one needs to closely monitor various parameters of the flow; and in this paper we also provide a list of fluid dynamic parameters and simple criterions that indicate adherence/nonadherence to expected flow regimes, and can be used to monitor the real field conditions. More broadly, our results suggest that high viscosity CFs are most suited for optimal cleaning of biofilms formed on pipeline walls. It is however noted that, high viscosity CFs require higher pumping power, and thus, in places where this is difficult to achieve, operating conditions for implementation with medium viscosity CFs have also been suggested. The method suggested is highly targeted and ensures minimal usage of cleaner-fluid.

\section{$\underline{\text { RESULTS }}$}

Considering that a cleaner-fluid (CF) would already have the chemical properties to remove the biofilms on the pipeline walls, the challenge is to ensure 'efficient' and 'sufficient' contact between the cleaner-fluid and the pipeline walls. This is clearly a fluid dynamics problem, since one particular flow regime would maximise the contact while another might not. Achieving a particular flow regime, depends on the relative fluid properties, which in this case is sewage water and cleaner-fluid. Sewage water is assumed to have properties close to water and is an invariable factor in all the combinations investigated. So the problem now depends on the cleaner-fluid property, and the single most dominant factor is its viscosity. Experimental data of liquid-liquid flows of low viscosity and high viscosity fluids is available. However, medium viscosity ranges are less explored. Thus, flow regimes achievable for medium viscosity is unknown. In this study, following the demonstration that the simulation convincingly captures the liquid-liquid flows at for both high and low viscosity CFs, the middle viscosity range was also simulated, to ensure completeness of data.

\section{Flow morphology}

Here, we present the flow morphology obtained using simulations for low, medium, and high viscosity CF cases. In some cases where experimental observations of the flow regimes are available, the simulation results have been compared with them. Tables 1 - 3 present the flow regimes obtained for low, medium and high viscosity cleaner-fluids respectively. It was observed that the flow patterns matched expected trend for most cases except when the water velocities (sewage) were low. In low water velocity cases, we know that, quantitatively, the average void fraction across a cross section is captured reasonably well by the simulation, and it the spatial distribution that is not captured accurately. This mismatch is attributed to the inability of the interfacial force models used in the CFD simulation, which are essentially empirical models, to provide the correct force quantities, when it falls below the model's applicability range. The exercise of tuning these models for improving flow regime predictions at low water velocity cases is not carried out here since water velocity in sewage lines 
tend to be of the medium to high velocity ranges. It is observed from the simulations that two major parameters govern flow patterns: (a) the magnitude of superficial velocity ( $\left.s_{v e l}\right)$ of CF and water (low, medium, high) (b) their ratio $\mathrm{R}\left(\mathrm{R}=\mathrm{CF} s_{\text {vel }} /\right.$ water $\left.s_{\text {vel }} ; \mathrm{R}>1,=1,<1\right)$. Superficial velocity is a hypothetical flow velocity calculated as if the given phase or fluid were the only one flowing or present in a given cross sectional area. This is the common metric of reporting operating conditions in multiphase flows.

Based on the simulations conducted (Table 1-3), the following conclusions can be drawn,

1. For Low viscosity CFs:

- $\mathrm{R}<1$, and both $S_{\text {vel }}$ are low: CF is slower than water, thus CF plugs can be seen.

- $\mathrm{R}<1$, and both/only water's $S_{\text {vel }}$ is high: CF plugs form but have a deformed shape as water tends to exert higher force on the interfaces of plugs.

- R>1, and both $S_{v e l}$ are medium/low: Since CF is faster than water, it is able to retain its shape and larger plugs are seen

- R>1, and both $S_{v e l}$ are medium/high: intermittent long slugs that fill the entire pipeline can be seen.

2. For medium viscosity CFs:

- $\mathrm{R}<1$, and both $S_{\text {vel }}$ are low: larger plugs than previous case, as CF has higher viscosity and can retain shape better.

- $\mathrm{R}<1$, but water $S_{v e l}$ is medium: Though higher water velocity will tend to deform the plugs, the increased viscosity of $\mathrm{CF}$ enables it to counter this near the wall where the velocity of water is lower. Thus, water successfully disperses CF finely in the entire medium, but at the walls a fine CF film is formed.

- R>1, and both $S_{v e l}$ are low: water plugs instead of CF plugs since water has the lower velocity.

- R>1, and both $s_{\text {vel }}$ are medium/high: long slugs with wavy interface are formed.

3. For high viscosity CFs:

- $\mathrm{R}<1$, and both $S_{\text {vel }}$ are low: Due to high viscosity, CF interfaces remain intact closer to the walls. Thus, a coating of CF is constantly present at the wall. This is more pronounced at medium velocities of water

- R<1, but both $s_{\text {vel }}$ are medium/ high: Water disperses CF finely in the entire medium.

- R>1, and medium CF $S_{\text {vel }}$ with high water velocity: thin film of CF coats the walls.

- R>1, and both $S_{v e l}$ are medium/high: a mix of dispersion and slugs with wavy interface are seen

The understanding of how to apply these aforementioned observations will be discussed in the discussion section. 
Table 1: Comparison of Flow Patterns for Low viscosity CF and water flow

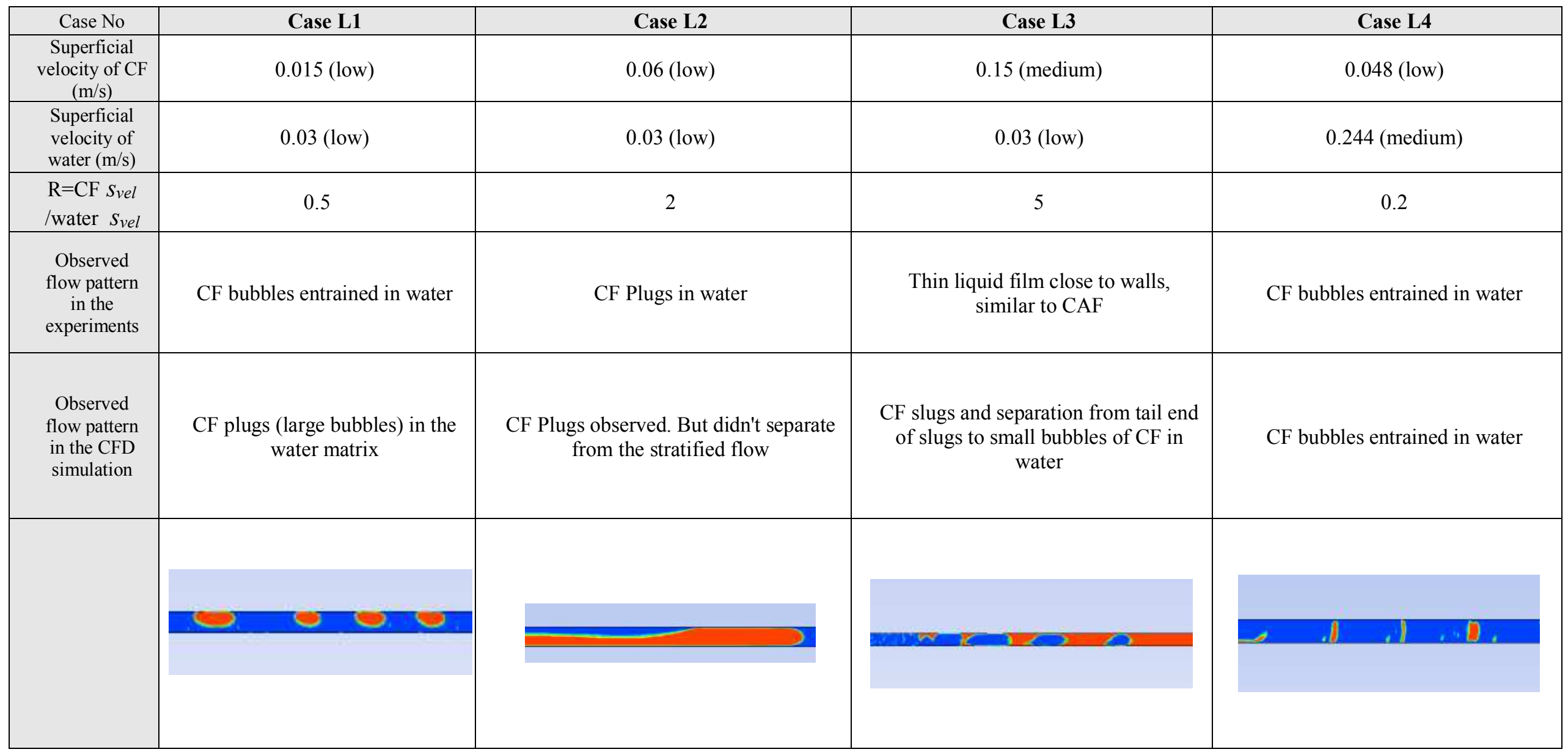


Table 1(contd): Comparison of Flow Patterns for Low viscosity CF and water flow

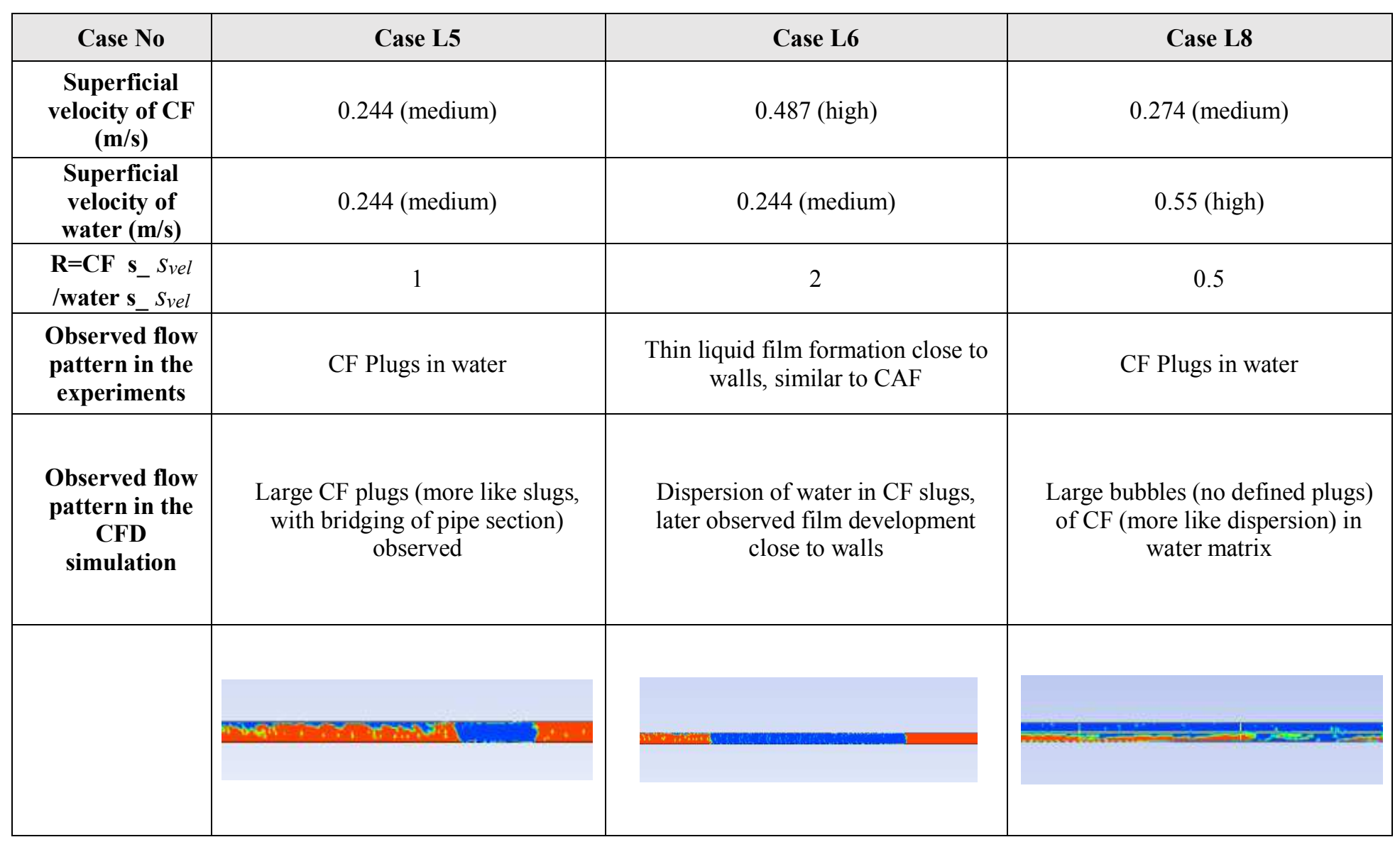


Table 2: Comparison of Flow Patterns for Medium viscosity CF and water flow

\begin{tabular}{|c|c|c|c|c|}
\hline Case No & Case M1 & Case M2 & Case M3 & Case M4 \\
\hline $\begin{array}{c}\text { Superficial velocity of } \\
\text { CF (m/s) }\end{array}$ & 0.015 (low) & $0.05(\mathrm{low})$ & 0.25 (medium) & 0.25 (medium) \\
\hline $\begin{array}{c}\text { Superficial velocity of } \\
\text { water (m/s) }\end{array}$ & $0.03(\mathrm{low})$ & 0.1 (medium) & $0.1($ medium) & 0.25 (medium) \\
\hline $\mathrm{R}=\mathrm{CF} \quad S_{\text {vel }} /$ water $S_{\text {vel }}$ & 0.5 & 0.5 & 2.5 & 1 \\
\hline $\begin{array}{c}\text { Observed flow pattern in } \\
\text { the CFD simulation }\end{array}$ & Plugs of CF in water & CAF & $\begin{array}{c}\text { Large amplitude wavy } \\
\text { stratified structures getting } \\
\text { transformed to dispersion }\end{array}$ & $\begin{array}{c}\text { Intermittent separated } \\
\text { flow, along with dispersion }\end{array}$ \\
\hline & & & & \\
\hline
\end{tabular}


Table 3: Comparison of Flow Patterns for High viscosity CF and water flow

\begin{tabular}{|c|c|c|c|c|c|c|}
\hline Case No & Case H1 & Case H2 & Case H4 & Case H6 & Case $\mathrm{H7}$ & Case H15 \\
\hline $\begin{array}{c}\text { Superficial } \\
\text { velocity of } \mathbf{C F} \\
(\mathbf{m} / \mathbf{s})\end{array}$ & 0.06 (low) & 0.1 (medium) & 0.12 (medium) & 0.12 (medium) & 0.12 (medium) & 0.4 (high) \\
\hline $\begin{array}{c}\text { Superficial } \\
\text { velocity of } \\
\text { water }(\mathrm{m} / \mathrm{s})\end{array}$ & 0.23 (medium) & 0.05 (low) & 0.18 (medium) & 0.61 (high) & 0.81 (high) & 0.4(high) \\
\hline $\begin{array}{ll}\mathbf{R}=\mathbf{C F} & S_{\text {vel }} \\
\text { /water } & S_{\text {vel }}\end{array}$ & 0.26 & 2 & 0.66 & 0.2 & 0.15 & 1 \\
\hline $\begin{array}{l}\text { Observed } \\
\text { flow pattern } \\
\text { in the } \\
\text { experiments }\end{array}$ & - & Inversion of flow & $\begin{array}{c}\text { Concentric Annular } \\
\text { flow }\end{array}$ & $\begin{array}{c}\text { Lumps of CF } \\
\text { dispersed in water }\end{array}$ & $\begin{array}{l}\text { Lumps of CF } \\
\text { dispersed in water }\end{array}$ & CAF \\
\hline $\begin{array}{c}\text { Observed } \\
\text { flow pattern } \\
\text { in the CFD } \\
\text { simulation }\end{array}$ & & $\begin{array}{c}\text { CAF flow, but an } \\
\text { appreciable } \\
\text { communication between } \\
\text { top and bottom films } \\
\text { through webby } \\
\text { structures, seeming like } \\
\text { a kind of entrapped } \\
\text { water plugs in the webs } \\
\text { of CF films }\end{array}$ & $\begin{array}{c}\text { Concentric Annular } \\
\text { Flow }\end{array}$ & $\begin{array}{l}\text { Thin wafer type films } \\
\text { for the CAF flow. } \\
\text { Later transformed to } \\
\text { CF dispersion }\end{array}$ & $\begin{array}{l}\text { Large wavy } \\
\text { stratified, with CF } \\
\text { dispersion }\end{array}$ & CAF \\
\hline & 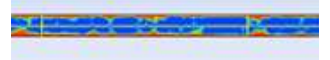 & 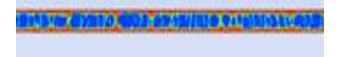 & 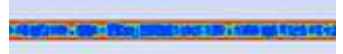 & 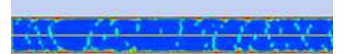 & 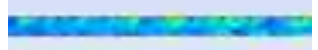 & parmanam \\
\hline
\end{tabular}




\section{Generic Trends of Macroscopic parameters}

Visual verification of flow regimes is not possible in sewage pipelines. Thus, it would be essential to monitor macroscopic parameters at various locations to verify the flow regime that is actually occurring. This section discusses the axial variation of pressure, $\mathrm{CF}$ fraction, and CF velocity.

\section{Pressure}

- It was observed that for a few lower viscosity cases, the injected CF does not retain its trajectory and eventually becomes a dispersed flow regime (e.g. L4 in Table 1). It was observed that at the location where the $\mathrm{CF}$ becomes dispersed, the pressure value in the pipeline dips towards the negative side, creating a vacuum pressure condition at the section (Fig 1a).

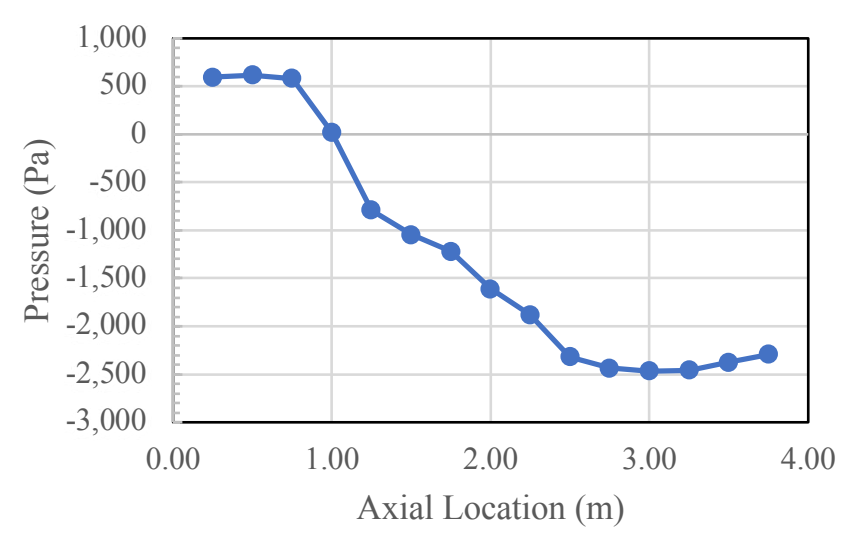

(a)

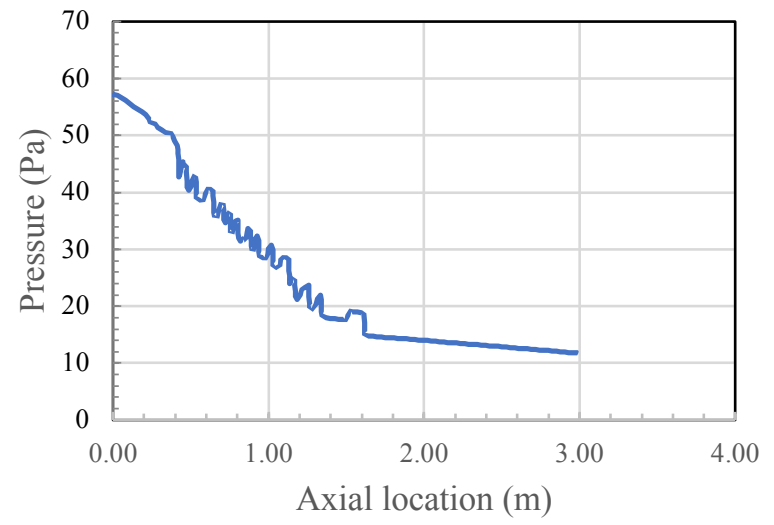

(b)

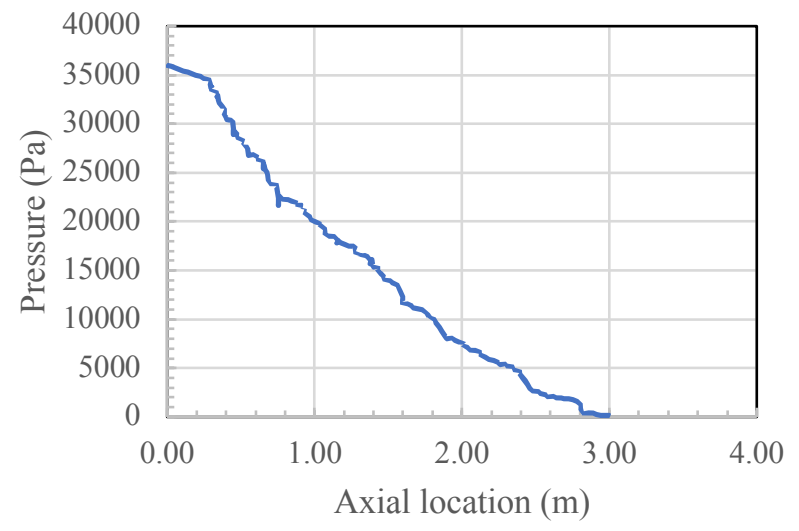

(c)

Fig. 1: (a) Axial variation of sectional averaged pressure for Case L4. The Pressure becomes negative indicating transition to a dispersed flow regime (b) Fluctuations observed in axial variation of linear (longitudinal central axis) pressure for Case L-1and is indicative of plug flow(b) Axial variation of linear (longitudinal central axis) pressure for Case H-1

- For cases L1 and H1, plots have been presented for comparison of variation of pressure along the central axis in the flow direction. (Fig 1 b,c). For the plug type flows as encountered in the case L1, the axial variation of pressure fluctuates suggesting a higher localized pressure due to the onset of plugs. 


\section{CF fraction and CF velocity}

For many of the cases it could be observed that the trends for CF fraction and CF velocity were morphologically similar.

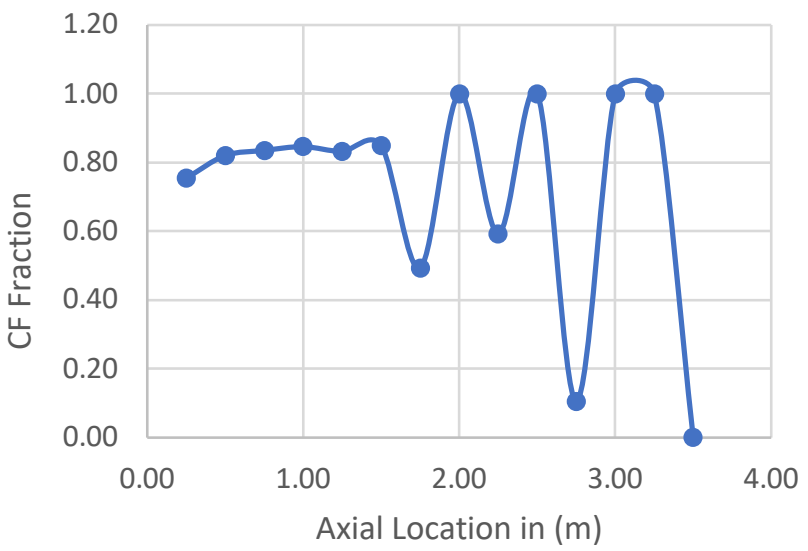

(a)

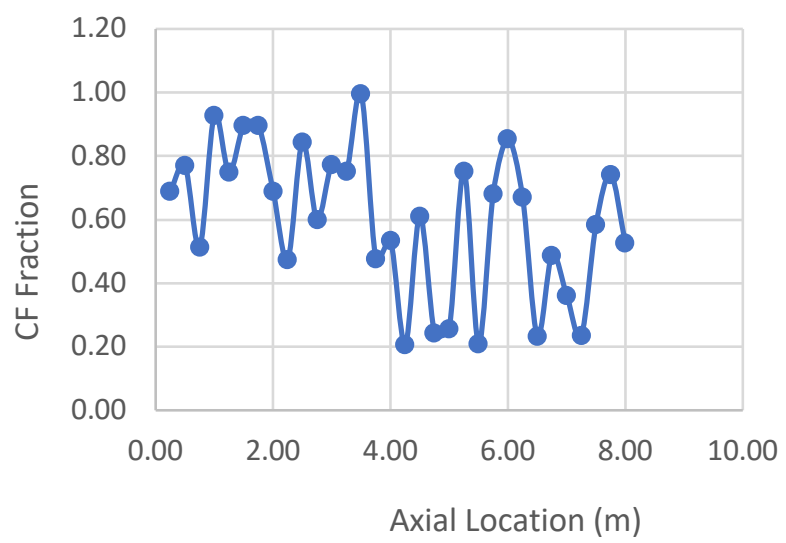

(c)

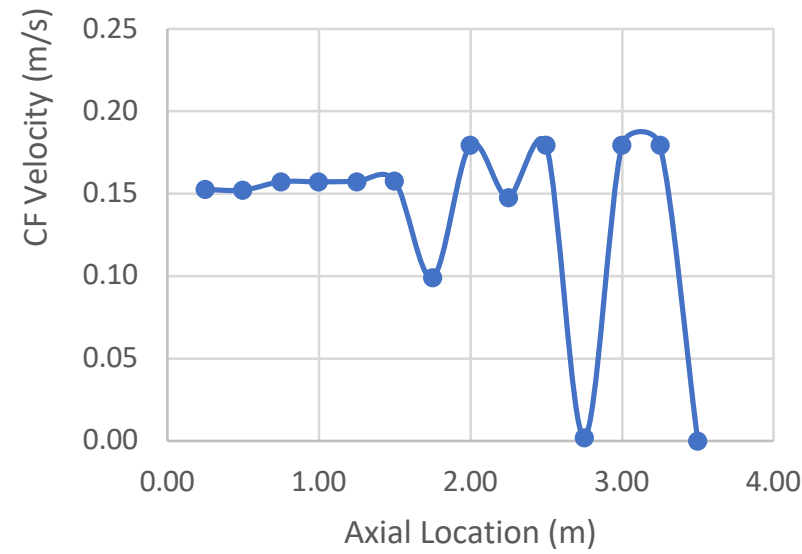

(b)

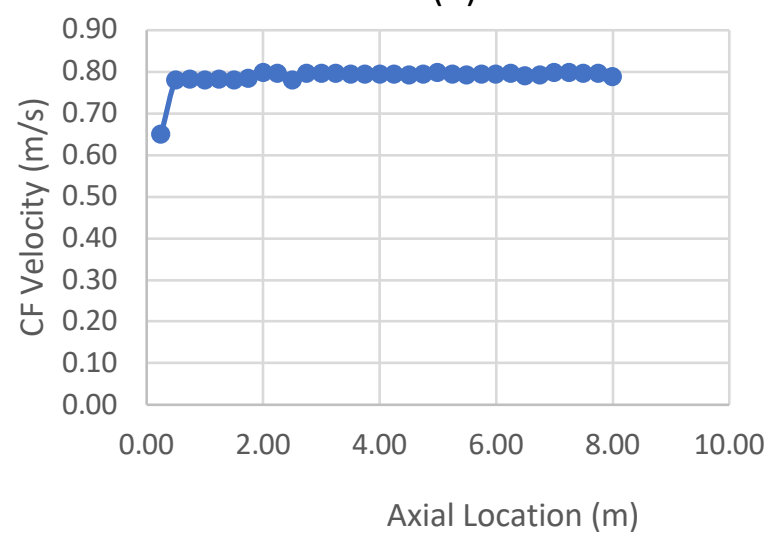

(d)

Fig 2: (a) Axial variation of sectional averaged CF fraction for Case L-3 (b) Axial variation of sectional averaged CF velocity for Case L-3. Large fluctuations in both (a) and (b) are indicative of large slugs in the pipeline (c) Axial variation of sectional averaged CF fraction for Case H-15 (d)Axial variation of sectional averaged CF velocity for Case H-15. Here, fluctuations in (c) and the absence of those in (d) is indicative of wavy stratified flow in the presence of smaller dispersed bubbles

- Severe fluctuation could be observed in the L3 case wherein slugs of CF was observed as the dominant flow feature. So generally speaking, the distribution of large bubble like plugs or slugs could produce severe fluctuations in the velocity of CF inside the domain. (Fig.2. a, b). 
- As the flow regimes transformed to the dispersion patterns, the velocity variations were observed to attain a quasi-stable state with little fluctuations. This implies that for a uniformly dispersed flow regime, the velocity fluctuations can be minimum although the absolute magnitude of velocities may be higher.

- Similar plots can be observed for the flow regime comprising of wavy stratified along with dispersion of CF in water. Here, although there was a considerable oscillation (owing to the impact of wavy stratified structures) in the volume fraction plot, the CF velocity stood nearly a constant in the axial direction, even though the value was higher than the inlet boundary condition velocity value. (Fig 2. c, d)

- Another significant observation was that, for higher inlet superficial velocities, the velocity values at different sections in the domain were reaching values higher than the inlet values. The larger velocities of CF plugs could be attributed to the lesser share of cross section available for flow leading to a nozzle like effect causing acceleration.

- Oscillatory nature of the volume fraction graphs with higher frequency and lower amplitude could also be correlated with bubbly flows while large amplitude small frequency oscillations of volume fraction graphs represented plug or slug flows.

\section{DISCUSSION}

Literature states that biofilms that grow in sewage pipelines are potential locations where COVID-19 like virus can aggregate. Through this work, we demonstrate that interfacial hydrodynamic concepts can be used to ensure removal of biofilms formed on pipeline walls. The method suggested is target specific i.e. it transports cleanerfluid right to the walls of the pipeline, but at the same time ensures effective usage of cleaner-fluid with minimal wastage.

First and foremost, the present study has tested the CFD technique of Multi-fluid VOF and has demonstrated that it is a reliable tool that can be used to predict different flow regime formations, for multiphase horizontal flows in pipelines. This method has been shown to have better accuracy when compared with other popular interface capturing multiphase techniques whenever a wide range of flow regimes need to be predicted. This is a one of a kind simulation catering to a range of fluid viscosity ranging right from high to low.

The main result of our numerical simulations is that we are able to recommend superficial velocities at which cleaner-fluids need to be pumped into the sewage line, so as to achieve flow regimes that ensure delivery of cleaner fluid to the periphery of the tube walls. Our simulations indicate that most effective cleaning can be achieved by using a cleaner-fluid with a high viscosity ( $\sim 5000 \mathrm{cP})$ ). In such cases, a low- medium velocity $(\sim 0.05-0.3 \mathrm{~m} / \mathrm{s})$ of CF and water would ensure that the cleaner fluids are in constant contact with the pipe walls (Fig. 3a). On the other hand, injecting cleaner fluids at very low superficial velocities $(\sim 0.03 \mathrm{~m} / \mathrm{s})$ is not 
recommended as the thickness of the film at the wall becomes very thin (Fig.3b); consequently, this should be used only when the pipes are either new or in cases where cleaning cycles are usually very frequent.

When using medium viscosity CFs $(\sim 600 \mathrm{cP})$, the flow configuration as shown in Fig. $3 \mathrm{c}$, can be achieved; but such a flow regime can be achieved only when injecting cleaner-fluid at medium superficial velocity into a pipeline where waste water is flowing at a high superficial velocity. This is a very narrow restrictive range of operation. Moreover, this flow pattern would require large volumes of $\mathrm{CF}$, since a lot of the cleaner fluid is also present in the core flow. This might be suitable for cleaning effluents from highly contaminated regions such as hospitals, where disinfecting the water in the core is also essential.

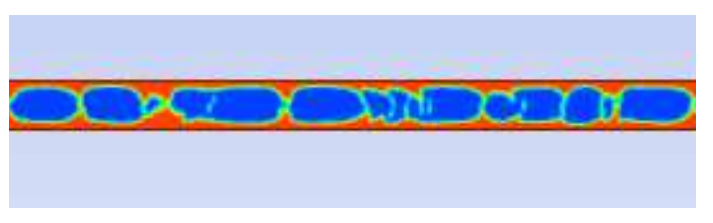

(a)

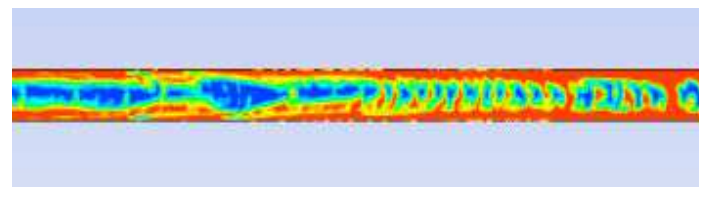

(c)

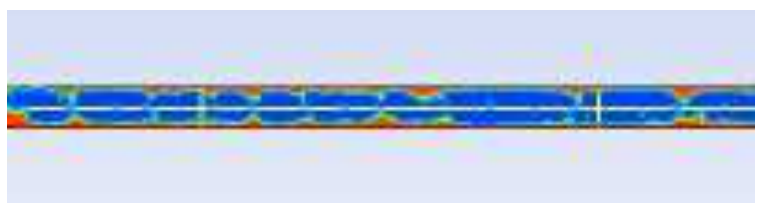

(b)

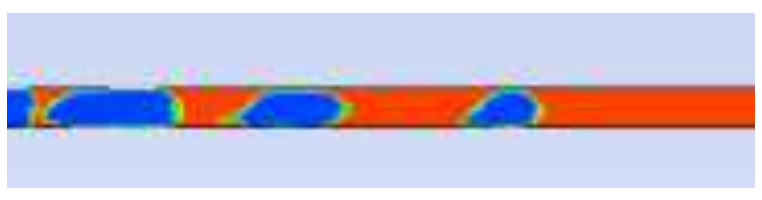

(d)

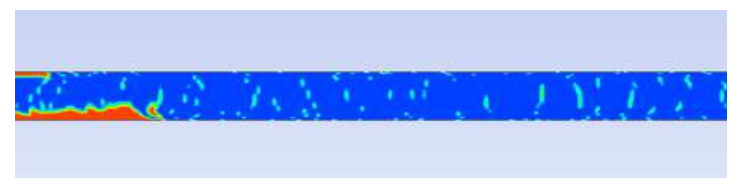

(e)

Fig 3: Flow pattern observed for (a) H-2 (b) H-1 (c) M-8 (d) L-3 (f) L-7. Red here is indicative of the cleaner fluid, while blue is indicative of the water effluent

When using low viscosity CFs $(\sim 16 \mathrm{cP})$, the best possible flow pattern that can be obtained is that of slug flow (Fig. 3d). This pattern is achievable when injecting cleaner-fluid at medium-high velocity into a pipe where the sewage water is moving at a low-medium velocity. In these cases, there is cyclic exposure of tube to waste water and cleaner-fluid. It also has to be pointed out that when using low viscosity cleaner -fluids, one must avoid 
injecting them at very low superficial velocities, especially when the waste water is flowing at a high velocity. This might occur inadvertently in monsoon seasons when the sewage flow is quite large and the usual injection velocity will be too low in comparison. The effect of the large difference in the relative velocity causes the cleaner-fluid to completely disperse into the medium and does not provide effective cleaning(Fig. 3e).

Real life conditions might not adhere to expected behaviour. Thus there is a need to closely monitor the flow occurring in the pipeline. Since flow regime visualisation is not possible in pipelines, measuring devices need to be used at regular intervals along the length of the pipeline to measure the macroscopic parameters' variation. The study also provides recommendations of what parameters to monitor on site to ensure adherence to expected patterns and can be applied in a wide range of conditions. Our numerical simulations show that pressure, cleaner fluid fraction, and its velocity are important indicators of the flow pattern existing within the pipe. Our simulations have also shown that the flow regimes that need to be avoided are dispersion regimes, and regimes where cleaner fluid is distributed in the medium in the form of bubbles, or plugs. Uneven stratification also is not desired since that exposes only one part of the pipeline circumference to the cleaner-fluid.

Based on our results, we have been able to identify the trends of macroscopic parameters which indicate that the aforementioned undesirable regimes are occurring. For example, If the average pressure at a cross-section were to change from positive to negative, anywhere along the pipeline, then it implies that the flow has transitioned into a dispersion regime. If, simultaneously, the CF fraction and velocity, have large oscillations then the flow is stratified. However, if the oscillation is small, then it indicates occurrence of bubbly flow regime. Slug and plug type flow regimes can be identified when the CF fraction and velocity values will fluctuate with large amplitude and smaller frequencies. In some slug and plug type flow regimes, cleaner fluid velocities might become larger than the inlet values anywhere along the length of the pipeline.

It is important to note that the theory represented here is based on certain important assumptions relating to the properties of the cleaner fluids. The foremost assumption made in this paper is that the density of the different CF fluids are same as that of water. This assumption is done on the basis of the physical requirement that we would want to prevent gravity driven stratification of CF. One way this can be achieved is by adding chemical additives such as carbon tetrachloride. Another important assumption has been made with regard to the surface tension of the CF. For this work, surface tension corresponding to oils with viscosity values same as the ones used in this study have been used. Other auxiliary assumptions are (i) chemical properties of the cleaner fluid and waste water is not significant enough to modify flow features (ii) Waste water is taken to have pure water properties (iii) Presence of solid and gas phases are assumed to have negligible effect on the flow (v)Large parts of the waste system are pipelines and not open channels; a valid assumption especially in cities. Our results, 
with the aforementioned specific CF fluid property combinations of surface tension, viscosity and density, have been tested over a range of operating conditions and since the results are presented in terms of superficial velocities, these can be extended to pipelines of different sizes and different velocities. One way to apply the findings of this study, is by using chemical additives similar to the ones suggested above, to ensure that the chosen CF has properties similar to the ones used in this study. Alternatively, the simulation methodology used this study can be quickly used, with a given set of properties of the $\mathrm{CF}$, and corresponding flow conditions that will ensure wetting of the walls by the CF, can be determined. Simulation using the method suggested in this paper will also greatly help in estimating flow behaviour when the geometry is scaled up and that too at a minimal cost. The validity of the theory discussed here can also be tested quite easily by conducting a preliminary test at any local municipal facility, house, building or hospital.

The importance of our result stems from the fact that it permits easy implementation in developing countries using minimal resources/equipment. This method also helps minimise cleaner fluid usage in order to restrict any environmental impacts. Our result have a wider range of applicability than just applying it to waste water treatment. Since, it is purely defined by fluid viscosity and fluid properties, it can be extended to address 'clean drinking water' problems. The solution proposed is very simple and can be implemented at every housing/ building and society level. Thus, our results offer an important new direction of research for interfacial hydrodynamics as a means for addressing different social problems at industrial levels. We offer an easy, affordable and readily implementable solution to curtail the outbreak of COVID 19.

\section{METHODS}

\section{Multi-fluid VOF: Two fluid model with interface capturing approach}

The coupled model in the Multi-fluid VOF solves an equation for the volume fraction in the computational cells similar to the VOF scheme, using the numerical approach of Geo-Reconstruct, compressive, and CICSAM interface capturing methods. This interface sharpening option along with the basic continuity, momentum and energy equations being solved for individual phases make an improved version of Eulerian method capable of capturing different flow regimes. [17]. ANSYS FLUENT commercial platform has been used to carry out the simulations

Generalized form of governing equations:

Continuity for $\mathrm{k}^{\text {th }}$ phase: $\quad \frac{\partial\left(\rho_{k} \alpha_{k}\right)}{\partial \mathrm{t}}+\nabla \cdot\left(\rho_{k} \alpha_{k} \vec{u}_{k}\right)=S_{\mathrm{k}}+\sum_{p=1}^{n}\left(\dot{m}_{p k}-\dot{m}_{k p}\right)$ 


$$
\frac{\partial \alpha_{k}}{\partial \mathrm{t}}+\vec{u}_{k} \cdot \nabla \alpha_{k}=0
$$

Momentum equation for $\mathrm{k}^{\text {th }}$ phase :

$$
\begin{aligned}
\frac{\partial\left(\rho_{k} \alpha_{k} \vec{u}_{k}\right)}{\partial \mathrm{t}}+\nabla \cdot\left(\rho_{k} \alpha_{k} \vec{u}_{k} \vec{u}_{k}\right) \\
=-\alpha_{k} \nabla \mathrm{p}+\nabla \cdot\left(\overline{\overline{\tau_{k}}}\right)+\rho_{k} \alpha_{k} \vec{g}+\sum_{p=1}^{n}\left(\vec{R}_{p k}+\dot{m}_{p k} \vec{u}_{p k}-\dot{m}_{k p} \vec{u}_{k p}\right)+\vec{F}_{\text {Drag, } k}+\vec{F}_{L i f t, k} \\
+\vec{F}_{\text {Virtual mass }, k}+\vec{F}_{\text {Turbulent dispersion }, k}
\end{aligned}
$$

$\overline{\overline{\tau_{k}}}$ is the $\mathrm{k}^{\text {th }}$ phase stress-strain tensor,

$\vec{R}_{p k}$ is an interaction force between phases and $\mathrm{p}$ is the pressure shared by phases.

Continuity equation for volume fraction is solved for individual phases. Considering $\mathrm{k}^{\text {th }}$ phase,

$$
\frac{1}{\rho_{k}}\left[\frac{\partial\left(\rho_{k} \alpha_{k}\right)}{\partial \mathrm{t}}+\nabla \cdot\left(\rho_{k} \alpha_{k} \vec{u}_{k}\right)=-S_{\alpha_{k}}+\sum_{p=1}^{n}\left(\dot{m}_{p k}-\dot{m}_{k p}\right)\right]
$$

For the primary phase, volume fraction is computed based on the equation: $\sum_{k=1}^{n} \alpha_{k}=1$

Shared momentum equation:

$$
\frac{\partial(\rho \vec{u})}{\partial \mathrm{t}}+\nabla \cdot(\rho \vec{u} \vec{u})=-\nabla \mathrm{p}+\nabla \cdot\left[\mu\left(\nabla \vec{u}+\nabla \vec{u}^{T}\right]+\rho \vec{g}+\vec{F}\right.
$$

Anisotropic drag law has been used for the basic Multi-fluid VOF model. Under this, the viscous drag formulation has been used for the simulations. Effective Drag Coefficient in the principal direction:

$$
\mathrm{K}_{\mathrm{p}}=(\text { Kvisc })_{\mathrm{p}} * \alpha_{\mathrm{i}} * \alpha_{\mathrm{j}}
$$

$\alpha_{i}$ and $\alpha_{j}$ being the volume fractions of the $i^{\text {th }}$ and $j^{\text {th }}$ phases respectively. The viscous drag component in the principal direction is given by

$$
(\text { Kvisc })_{\mathrm{p}}=\frac{\mu}{\mathrm{l}_{\mathrm{c}}^{2}} \lambda_{\mathrm{p}}
$$

$\lambda_{\mathrm{p}}$ is the friction factor vector given in the principal direction. $l_{\mathrm{c}}$ is the length scale. $\mu=\mu_{\mathrm{i}} \alpha_{\mathrm{i}}+\mu_{\mathrm{j}} \alpha_{\mathrm{j}}$, the mixture viscosity. The surface tension force is estimated as a Continuum Surface Force (CSF), following the work of [18]. An additional source term is appeared in the momentum equation on implementing this model for accommodating the surface tension force in the VOF calculations at the interface. Same/similar densities of bot 
liquids-liquids since we want to prevent stratification based segregation. Other effects considered negligible in this case is wettability effects with the tube wall.

\section{Procedure of Analysis}

Shi (2015)[19] has considered a T-junction tubular domain with dual inlets- one each for the CF and water fluids (CF in the horizontal branch and water in the vertical branch). Each branch is having $200 \mathrm{~mm}$ length and axial length extending up-to 8 meters downstream of the meeting junction. The simulation has been setup based on this experimental case with the validation carried out against the data presented in this paper. A 2D approximation was employed. A 2D domain was deemed sufficient since literature states that flow evolution is more dominated by events in the axial flow direction rather than the events in the transverse direction. The metering of most hydrodynamic parameters in the experiment being carried out in an 'averaged' approach, the 2D cases can present with equivalence for comparative validation without loss of generality. More Details of simulation setup has been provided in supplement.

The study has been performed for three different fluid systems consisting of three different CF viscosities- low, medium and high(Table 4). This is being done to compare the flow evolution features (including the regime of flow and other quantitative flow parameters like velocities of individual phases, etc.) as the CF viscosity varies. Table shows the different cases considered including the boundary conditions (superficial velocities of CF and water), multiphase model chosen and the viscosity of the CF selected. Mesh independence is discussed in the supplement. Based on this study the remaining simulations will be done using Mesh-3 with a total of 3.89 lakh hexahedral elements which is considered as the optimum one. Details of other simulation settings, grid independence, validation against macroscopic parameters, are provided in the supplement document. 
Table 4: Different cases showing the range of CF viscosities, velocity ranges used for computational analysis

\begin{tabular}{|c|c|c|c|c|}
\hline Case No & $\begin{array}{l}\text { Viscosity of } \\
\text { CF (cP) }\end{array}$ & Multiphase model & $\begin{array}{l}\text { Superficial } \\
\text { velocity of } \mathrm{CF} \\
(\mathrm{m} / \mathrm{s})\end{array}$ & $\begin{array}{l}\text { Superficial velocity of water } \\
(\mathrm{m} / \mathrm{s})\end{array}$ \\
\hline L-1 & 16.8 & Multi-fluid VOF & 0.015 & 0.03 \\
\hline L-2 & 16.8 & Multi-fluid VOF & 0.06 & 0.03 \\
\hline L-3 & 16.8 & Multi-fluid VOF & 0.15 & 0.03 \\
\hline L-4 & 16.8 & Multi-fluid VOF & 0.048 & 0.244 \\
\hline $\mathrm{L}-5$ & 16.8 & Multi-fluid VOF & 0.244 & 0.244 \\
\hline L-6 & 16.8 & Multi-fluid VOF & 0.487 & 0.244 \\
\hline L-7 & 16.8 & Multi-fluid VOF & 0.055 & 0.55 \\
\hline L-8 & 16.8 & Multi-fluid VOF & 0.274 & 0.55 \\
\hline L-9 & 16.8 & Multi-fluid VOF & 0.55 & 0.55 \\
\hline $\mathrm{L}-10$ & 16.8 & Multi-fluid VOF & 1.1 & 0.55 \\
\hline $\mathrm{H}-1$ & 5000 & Multi-fluid VOF, transient & 0.06 & 0.23 \\
\hline $\mathrm{H}-2$ & 5000 & Multi-fluid VOF & 0.10 & 0.05 \\
\hline $\mathrm{H}-4$ & 5000 & Multi-fluid VOF & 0.12 & 0.18 \\
\hline H-6 & 5000 & Multi-fluid VOF & 0.12 & 0.61 \\
\hline $\mathrm{H}-7$ & 5000 & Multi-fluid VOF, steady & 0.12 & 0.81 \\
\hline $\mathrm{H}-15$ & 5000 & Multi-fluid VOF & 0.40 & 0.40 \\
\hline H-17 & 5000 & Multi-fluid VOF & 0.40 & 0.80 \\
\hline M-1 & 612 & Multi-fluid VOF & 0.015 & 0.03 \\
\hline M-2 & 612 & Multi-fluid VOF & 0.05 & 0.1 \\
\hline M-3 & 612 & Multi-fluid VOF & 0.25 & 0.1 \\
\hline M-4 & 612 & Multi-fluid VOF & 0.25 & 0.25 \\
\hline M-8 & 612 & Multi-fluid VOF & 0.274 & 0.55 \\
\hline M-9 & 612 & Multi-fluid VOF & 0.06 & 0.03 \\
\hline
\end{tabular}


Nomenclature

\begin{tabular}{|c|c|c|c|}
\hline \multicolumn{2}{|r|}{ General Symbols } & \multicolumn{2}{|r|}{ Greek Symbols } \\
\hline Symbol & Particular & Symbol & Particular \\
\hline $\mathrm{G}_{\mathrm{g} / \mathrm{l}}$ & $\begin{array}{l}\text { Mass flux of individual phase } \\
\text { (gas or liquid ) }\end{array}$ & $\alpha_{k}$ & $\begin{array}{l}\text { Volume fraction of component } \\
\text { ' } \mathrm{k} \text { ' }\end{array}$ \\
\hline $\mathrm{m}_{\mathrm{pq}}$ & $\begin{array}{l}\text { Mass transfer from phase ' } \mathrm{p} \text { ' to } \\
\qquad \text { ' } \mathrm{q} \text { ' }\end{array}$ & $\beta_{\mathrm{N}}$ & $\begin{array}{l}\text { Volumetric quality of } \\
\text { component ' } N \text { ' }\end{array}$ \\
\hline $\mathrm{x}_{\mathrm{N}}$ & Mass fraction of component ' $\mathrm{N}$ ' & $\chi_{\mathrm{N}}$ & Mass quality of component ' $N$ ' \\
\hline $\mathrm{U}_{\mathrm{s} \text {,air }}$ & Superficial air velocity & $\rho_{\mathrm{k}}$ & Density of phase ' $k$ ' \\
\hline $\mathrm{U}_{\mathrm{s}, \mathrm{oil}}$ & Superficial oil velocity & $\mu_{\mathrm{k}}$ & Viscosity of the component ' $\mathrm{k}$ ' \\
\hline $\mathrm{u}_{\mathrm{Nji}}$ & $\begin{array}{l}\text { Drift velocity of component ' } \mathrm{N} \text { ' } \\
\text { in the direction ' } \mathrm{I} \text { ' }\end{array}$ & $\gamma_{\mathrm{i}, \mathrm{j}}$ & Local dispersion function \\
\hline $\mathrm{j}_{\mathrm{Nji}}$ & $\begin{array}{l}\text { Drift flux of component ' } N \text { ' in } \\
\text { the frame of reference moving } \\
\text { at } \mathrm{j}_{\mathrm{i}}\end{array}$ & $\tau$ & Shear stress in the fluid field \\
\hline $\mathrm{f}_{\mathrm{s}}$ & Slug frequency & $\sigma$ & Surface tension coefficient \\
\hline $\mathrm{L}_{\mathrm{s}}$ & Slug length & & \\
\hline $\mathrm{U}_{\mathrm{SL}} \& \mathrm{U}_{\mathrm{SG}}$ & Velocity of liquid and gas slugs & & \\
\hline $\mathrm{u}_{\mathrm{Lf}}$ & $\begin{array}{l}\text { Velocity of liquid in the film } \\
\text { region of slug flow }\end{array}$ & & \\
\hline $\overrightarrow{\mathrm{u}}_{\mathrm{k}}$ & $\begin{array}{c}\text { Instantaneous velocity vector of } \\
\text { phase ' } k \text { ' }\end{array}$ & & \\
\hline$C_{D}$ & Drag coefficient & & \\
\hline$a_{s}$ & Interaction area density & & \\
\hline $\operatorname{Re}$ & Reynolds No & & \\
\hline
\end{tabular}

Note: All the other symbols have been defined and used as per the context. 


\section{$\underline{\text { References }}$}

1. WHO Global, 2020, Water, sanitation, hygiene, and waste management for the COVID-19 virus: interim guidance, WHO/2019-nCoV/IPC_WASH/2020.3.https://www.who.int/publications/i/item/watersanitation-hygiene-and-waste-management-for-covid-19 (2020)

2. Lakshmi, 2020, Coronavirus Metrowater tests show prevalence of viral RNA in sewage collected from Chennai, The Hindu, https://www.thehindu.com/news/cities/chennai/coronavirus-metrowater-testsshow-prevalence-of-viral-rna-in-sewage-collected-from-chennai/article31485182.ece (2020)

3. Naddeo, V., and Liu, H.,. Editorial Perspectives: 2019 novel coronavirus (SARS-CoV-2): what is its fate in urban water cycle and how can the water research community respond? Environmental Science: Water Research \& Technology, 2020; DOI: 10.1039/d0ew90015j

4. B.Tomlinson, C. Cockram, 2003, SARS: experience at Prince of Wales Hospital, VOLUME 361, ISSUE 9368, P1486-1487, MAY 03, 2003 .DOI:https://doi.org/10.1016/S0140-6736(03)13218-7,

5. P. M. Gundy, C. P. Gerba, and I. L. 2008. Survival of Coronaviruses in Water and Wastewater . Food Environment Virology 2009;1(1):10-4. http://dx.doi.org/10.1007/s12560-008-9001-6

6. S. Chan, K. Pullerits, A. Keucken, K. M. Persson, C.J. Paul \& Peter Bacterial release from pipe biofilm in a full-scale drinking water distribution system, npj Biofilms and Microbiomes volume 5, Article number: 9 (2019)

7. U.Simunič, P.Pipp, M. Dular, and D.Stopar, The limitations of hydrodynamic removal of biofilms from the dead-ends in a model drinking water distribution system. Water Research,Volume 178, 1 July 2020, 115838

8. W. Li,T. Zheng, Y.Mac and J. Liu, Fungi characteristics of biofilms from sewage and greywater in small diameter gravity sewers. Environ. Sci.: Water Res. Technol., 6 (2020), pp. 532-539

9. X.Liu, B. Tang, Q. Gu, and X. Yu, Elimination of the formation of biofilm in industrial pipes using enzyme cleaning technique. MethodsX, Volume 1, 2014, Pages 130-136

10. P. Stiefel, S. Mauerhofer, J. Schneider, K. Maniura-Weber, U. Rosenberg, Q.Ren, Enzymes Enhance Biofilm Removal Efficiency of Cleaners. Antimicrob. Agents Chemother. 60, 3647-3652. doi: 10.1128/AAC.00400-16

11. Cerne, G., Petelin, S., Tiselj, I., 2001, Coupling of the interface tracking and the two fluid models for the simulation of incompressible two-phase flow. J. Comput. Phys., 171 (2001), pp. 776-804

12. G. Tryggvason et al., 2001, A Front tracking method for the computations of multiphase flows, Journal of Computational Physics, Volume 169, Issue 2, 20 May 2001, Pages 708-759

13. Kai Yan, D.F Che, 2009, A coupled model for simulation of the gas-liquid two-phase flow with complex flow patterns. A coupled model for simulation of the gas-liquid two-phase flow with complex flow patterns 
14. Frank,T., 2005, Numerical Simulation of Slug Flow Regime for an Air-Water two phase flow in horizontal pipes. In: The 11th International Topical Meeting on Nuclear Reactor Thermal-Hydraulics (NURETH11). Avignon, France.

15. Höhne, T., Vallée, C., 2010. Experiments and numerical simulations of horizontal two-phase flow regimes using an interfacial area density model. J. Comput. Multiphase Flows, 2 (2010), pp. 131-143

16. Bartosiewicz, Y., Seynhaeve, J.-M., Vallée, C., Höhne, T., Laviéville, J., 2010. Modeling free surface flows relevant to a PTS scenario: comparison between experimental data and three RANS based CFD codes. Comments on the CFD-experiment integration and best practice guideline. Nucl. Eng. Des., 240 (2010), pp. 2375-2381

17. ANSYS-FLUENT R14.0, Theory Guide, 2011

18. J.U. Brackbill , D.B. Kothe, C. C. Zemach ,1992, A continuum method for modelling surface tension. J. Comput. Phys., 100 (1992), pp. 335-354

19. Shi J., A Study On High-Viscosity Oil-Water Two-Phase Flow In Horizontal Pipes, Cranfield University, PhD Academic Year: $2012-2015$.

20. Charles et al., 1961, The Horizontal Pipeline Flow of Equal Density Oil-Water Mixtures. The Canadian Journal of Chemical Engineering, February, 1961 , pp. 27-36

\section{Affiliations}

Both authors are from Department of Mechanical Engineering, Indian Institute of Technology Bombay, Mumbai 400076, India

\section{Contributions}

P.P.S. and J.S.M conceived the research problem. P.P.S performed and analysed the results. P.P.S and J.S.M interpreted the results, and wrote the manuscript. All authors reviewed the manuscript and agreed with the final version.

\section{Corresponding author}

Correspondence to Janani Srree Murallidharan

\section{Ethics declarations}

Competing interests: The authors declare no competing interests. 
Figures

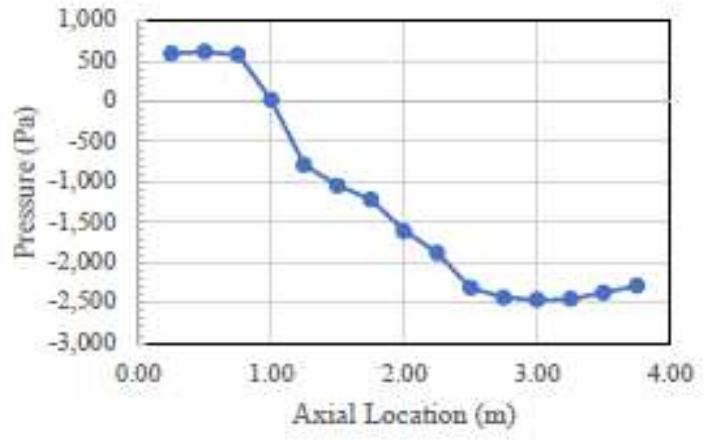

(a)

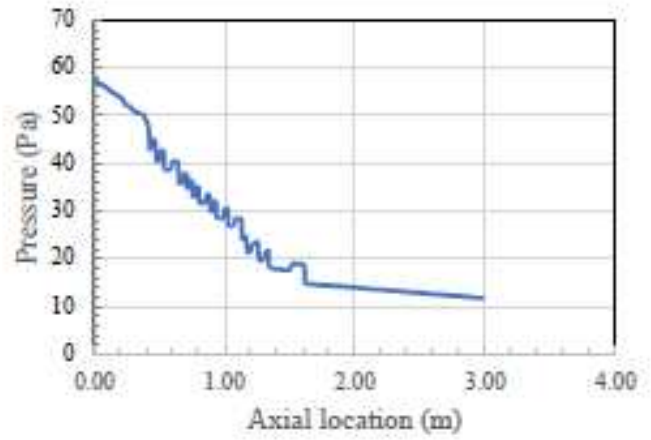

(b)

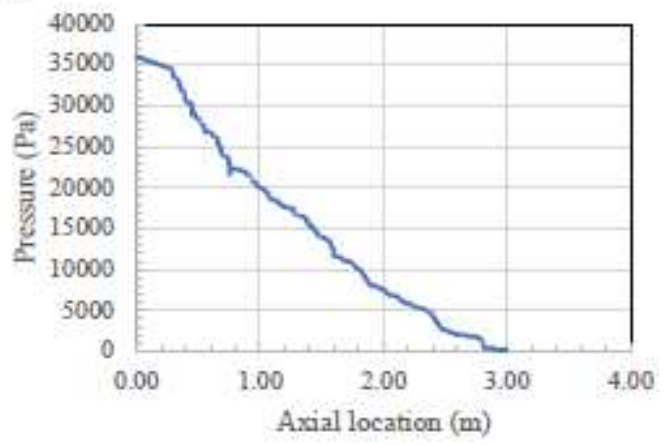

(c)

\section{Figure 1}

(a) Axial variation of sectional averaged pressure for Case L4. The Pressure becomes negative indicating transition to a dispersed flow regime (b) Fluctuations observed in axial variation of linear (longitudinal central axis) pressure for Case L-1 and is indicative of plug flow(b) Axial variation of linear (longitudinal central axis) pressure for Case $\mathrm{H}-1$ 


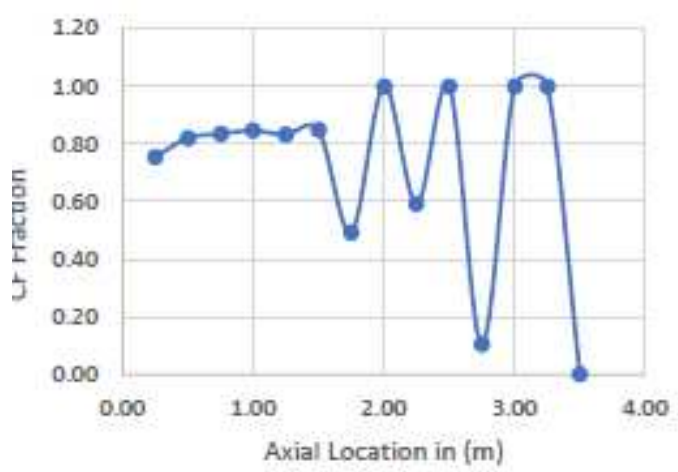

(a)

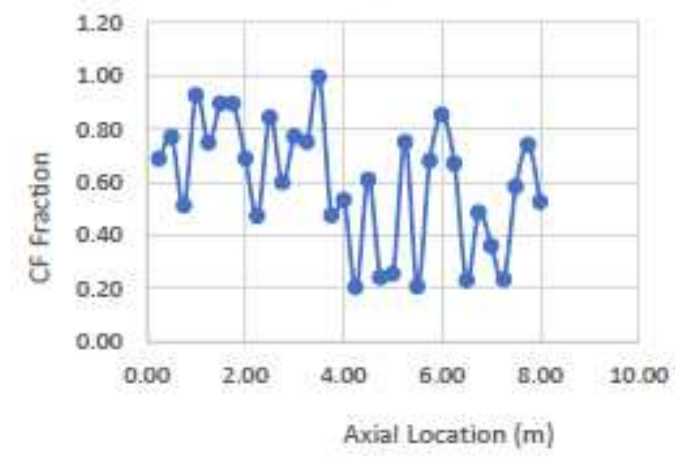

(c)

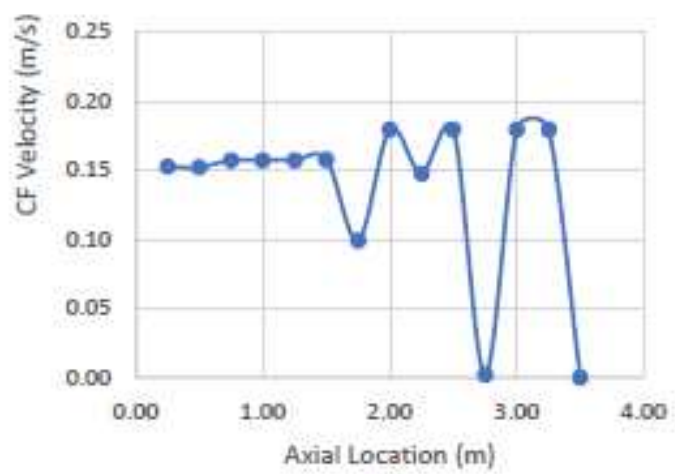

(b)

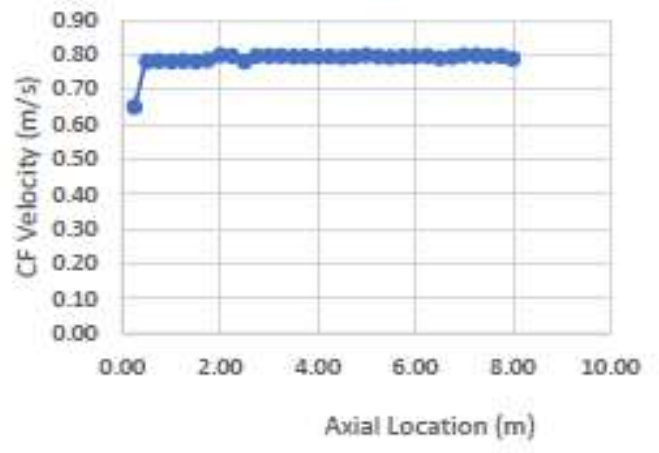

(d)

\section{Figure 2}

(a) Axial variation of sectional averaged CF fraction for Case L-3 (b) Axial variation of sectional averaged CF velocity for Case L-3. Large fluctuations in both (a) and (b) are indicative of large slugs in the pipeline (c) Axial variation of sectional averaged $\mathrm{CF}$ fraction for Case $\mathrm{H}-15$ (d)Axial variation of sectional averaged CF velocity for Case H-15. Here, fluctuations in (c) and the absence of those in (d) is indicative of wavy stratified flow in the presence of smaller dispersed bubbles
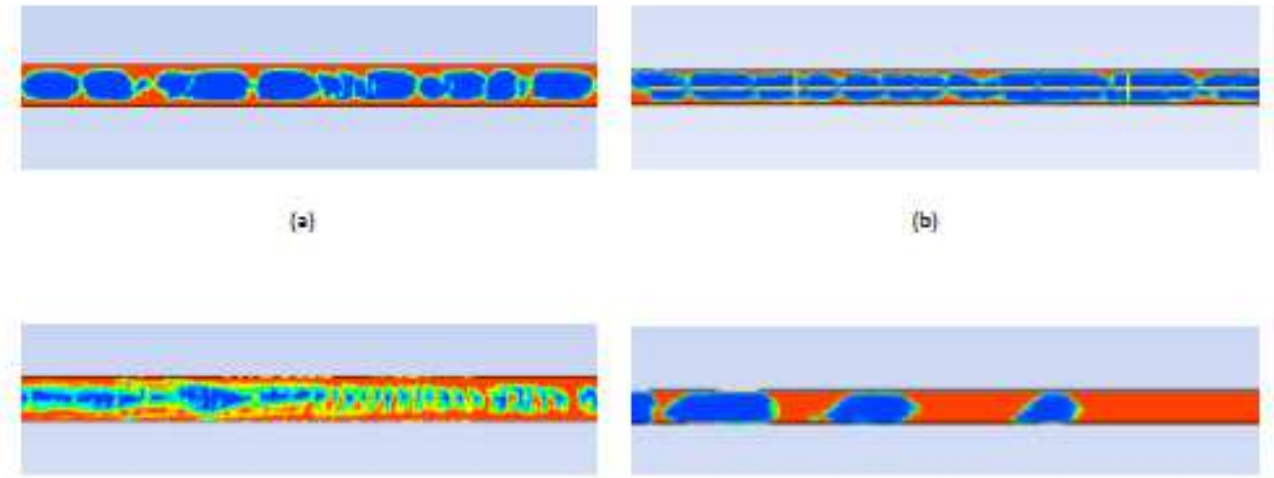

(c)

(c)

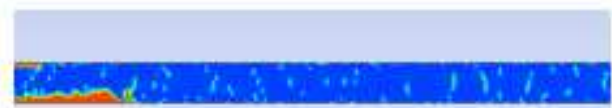


Figure 3

Flow pattern observed for (a) H-2 (b) H-1 (c) M-8 (d) L-3 (f) L-7. Red here is indicative of the cleaner fluid, while blue is indicative of the water effluent

\section{Supplementary Files}

This is a list of supplementary files associated with this preprint. Click to download.

- SupplementaryMaterial.pdf 